\title{
Erratum to: Modeling of the Austenitization of Ultra-high Strength Steel with Cellular Automation Method
}

BIN ZHU, YISHENG ZHANG, CHAO WANG, PEI XING LIU, WEI KANG LIANG, and JIAN LI

DOI: $10.1007 / \mathrm{s} 11661-014-2327-9$

(C) The Minerals, Metals \& Materials Society and ASM International 2014

Erratum to: METALLURGICAL AND MATERIALS

TRANSACTIONS A

DOI 10.1007/s11661-014-2255-8

THE following are corrections to the original article:

Page 1, Abstract, line 10: $10 \mathrm{~K}\left(-263{ }^{\circ} \mathrm{C}\right) / \mathrm{s}$ should be $10 \mathrm{~K}\left(10{ }^{\circ} \mathrm{C}\right) / \mathrm{s}$.

Page 4, Fig. 2 caption: $10 \mathrm{~K}\left(794.1^{\circ} \mathrm{C}\right) / \mathrm{s}$ should be $10 \mathrm{~K}\left(10^{\circ} \mathrm{C}\right) / \mathrm{s}$.

Page 5, IV. Procedure of Simulation, second paragraph: $10 \mathrm{~K}\left(-263{ }^{\circ} \mathrm{C}\right) / \mathrm{s}$ should be $10 \mathrm{~K}\left(10{ }^{\circ} \mathrm{C}\right) / \mathrm{s}$.

Page 7, last paragraph: $10 \mathrm{~K}\left(-263^{\circ} \mathrm{C}\right) / \mathrm{s}$ should be $10 \mathrm{~K}\left(10^{\circ} \mathrm{C}\right) / \mathrm{s}$.

Page 10, VI. Impact of the Grain Size on Strain-Stress Curve, first paragraph: $10 \mathrm{~K}\left(-263{ }^{\circ} \mathrm{C}\right) / \mathrm{s}$ should be $10 \mathrm{~K}\left(10^{\circ} \mathrm{C}\right) / \mathrm{s}$; and $35 \mathrm{~K}\left(-238^{\circ} \mathrm{C}\right) / \mathrm{s}$ should be $35 \mathrm{~K}$ $\left(35^{\circ} \mathrm{C}\right) / \mathrm{s}$.

Page 10, VII. Summary and Conclusions, second paragraph: $10 \mathrm{~K}\left(-263{ }^{\circ} \mathrm{C}\right) / \mathrm{s}$ should be $10 \mathrm{~K}\left(10^{\circ} \mathrm{C}\right) / \mathrm{s}$.

BIN ZHU, Post-doctor, YISHENG ZHANG and JIAN LI, Professors, and CHAO WANG, PEI XING LIU, and WEI KANG LIANG, PhD Candidates, are with the State Key Lab of Materials Processing and Die \& Mold Technology, Huazhong University of Science and Technology, Wuhan 430074, P. R. China. Contact e-mail: zhangys@mail.hust.edu.cn

The online version of the original article can be found under doi: 10.1007/s11661-014-2255-8.

Article published online May 21, 2014 